\title{
Crystallinity-Dependent Thermoelectric Properties of a Two-Dimensional Coordination Polymer: $\mathrm{Ni}_{3}(2,3,6,7,10,11 \text {-hexaiminotriphenylene })_{2}$
}

\author{
Yoshiyuki Nonoguchi ${ }^{1,2, *}$, Dai Sato ${ }^{3}$ and Tsuyoshi Kawai ${ }^{1, *}$ \\ 1 Division of Materials Science, Nara Institute of Science and Technology, Ikoma 630-0192, Japan \\ 2 JST PRESTO, Kawaguchi 332-0012, Japan \\ 3 Graduate School of Materials Science, Nara Institute of Science and Technology, Ikoma 630-0192, Japan; \\ dai.sato@trichemical.com \\ * $\quad$ Correspondence: nonoguchi@ms.naist.jp (Y.N.); tkawai@ms.naist.jp (T.K.); Tel.: +81-743-72-6181 (Y.N. \& T.K.)
}

Received: 5 August 2018; Accepted: 29 August 2018; Published: 31 August 2018

Abstract: The evaluation of thermoelectric properties has recently become a standard method for revealing the electronic properties of conducting polymers. Herein we report on the thermoelectric properties of a two-dimensional coordination polymer pellets. The pellets of $\mathrm{Ni}_{3}(2,3,6,7,10,11 \text {-hexaiminotriphenylene })_{2}$, which has recently been developed, show n-type thermoelectric transport, dependent on crystallinity. The present results provide systematic feedback to the guideline for high-performance molecular thermoelectric materials.

Keywords: coordination polymers; thermoelectric properties; molecular conductors

\section{Introduction}

Due to the rapid progress of synthesis technology and increased interests in unique transport and thermoelectric properties of atomically thin two-dimensional materials, these have recently been explored. Such two-dimensional films typically consist of $\mathrm{sp}^{2}$ carbon (graphenes), black phosphorous, and transition metal dichalcogenides (TMDC) as well as typical oxide semiconductors [1]. Thermoelectric properties reflect the electronic structure around the Fermi level, which has been used for the investigation of transport phenomena. In this context, low-dimensional materials having unique electronic structures are preferred objects for thermoelectric studies.

Low-dimensional materials can be designed by organic and inorganic synthetic chemistry. Representative examples include charge transfer complexes whose electronic properties vary from molecular metals to semiconductors [2-4]. In these previous works, thermoelectric properties were evaluated mostly for determining carrier polarity and estimating band structures around the Fermi level. Recently, several groups have reported that one-dimensional metal coordination polymers (CPs) exhibit excellent thermoelectric properties. The 1,1,2,2-ethenetetrathiolate (ett)-containing polymers, poly $\left[\mathrm{Na}_{x}(\mathrm{Ni}\right.$-ett $\left.)\right]$ and poly $\left[\mathrm{Cu}_{x}(\mathrm{Cu}\right.$-ett $\left.)\right]$, showed n-type and p-type thermoelectric properties with the power factor of ca. 30 , and $15 \mu \mathrm{W} \cdot \mathrm{m}^{-1} \cdot \mathrm{K}^{-2}$, respectively [5]. This report was based on the discovery of high conductivity CPs.

One-dimensional CPs based on benzene tetraamine (BTA) are reported to exhibit moderate electrical conductivity, which has recently been used for application in the fabrication of a resistive random access memory [6]. Recently, conducting two-dimensional (2D-) CPs have been synthesized, which stimulates their electron transfer and transport studies. The components of framework determine their electronic and structural properties such as bandgap, and porosity. Among them, several 2D-CPs seem to show metallic conduction. Particularly, the small crystalline sheet of $\mathrm{Ni}_{3}(\mathrm{HITP})_{2}$ 
(HITP $=2,3,6,7,10,11$-hexaiminotriphenylene) shows the relatively high conductivity of $40 \mathrm{~S} \cdot \mathrm{cm}^{-1}$ [7] and has been proposed to act as an electrocatalyst [8]. The high conductivity in these studies relies on the formation of a stable radical form (Figure 1a). So far, the transport properties of $\mathrm{Ni}_{3}(\mathrm{HITP})_{2}$ have been yet unexplored well. In this work, we examine the thermoelectric properties of $\mathrm{Ni}_{3}(\mathrm{HITP})_{2}$ pellets prepared using several Ni salts. We reveal that all these pellets show the negative and small Seebeck coefficient, that is, n-type metallic characteristics. Additionally, the conductivity is dependent on the crystallinity determined using X-ray diffraction (XRD) patterns. These results suggest the $\mathrm{Ni}_{3}(\mathrm{HITP})_{2}$ is intrinsically a molecular metal, and support the validity to use it as various electron-transporting components including catalyst.

(a)

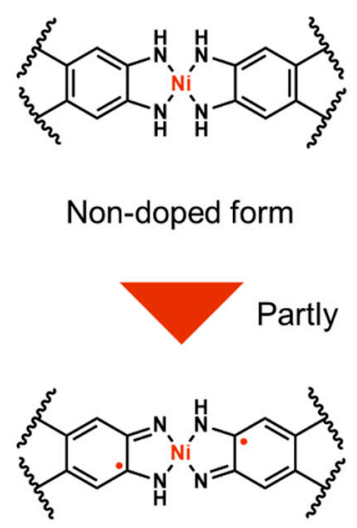

Radical form (b)

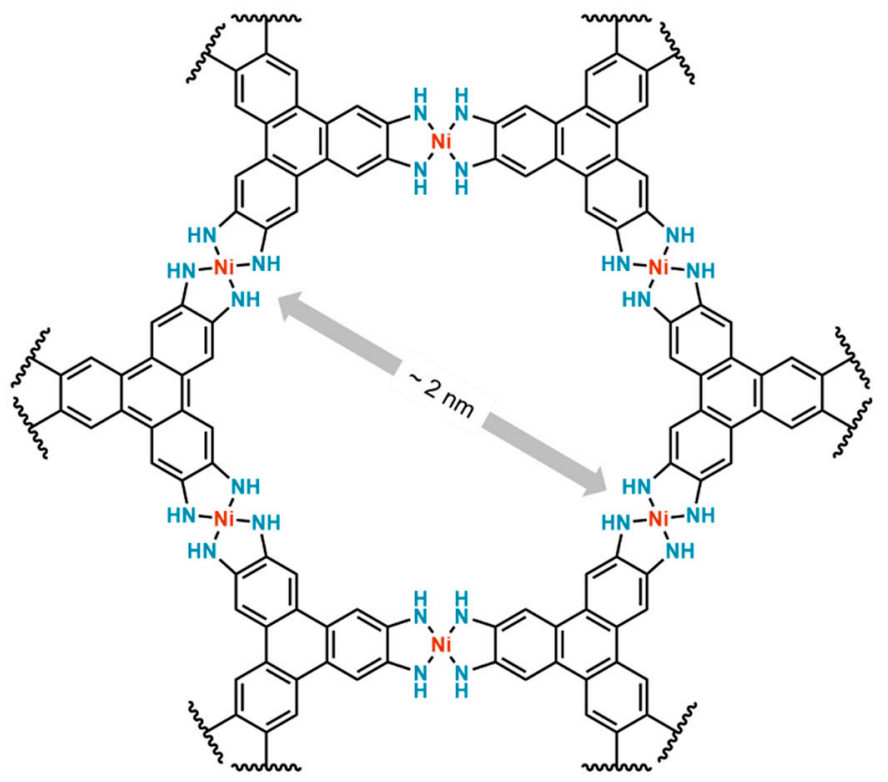

Figure 1. (a) The formation of the radical state that generates charged carriers. (b) The representative fragment of $\mathrm{Ni}_{3}(\mathrm{HITP})_{2}$.

\section{Materials and Methods}

We synthesized $\mathrm{Ni}_{3}(\mathrm{HITP})_{2}$ by following reported procedures (Scheme 1) [7], through the preparation of each intermediates $(2-4)[9,10]$. After the bromination of triphenylene [9], we synthesized the intermediate 3 (84\%, MALDI-TOF MS $m / z$ 1303.4; ${ }^{1} \mathrm{H}$ NMR (300 MHz, DMSO) 87.11-7.13 (m, 12H), $7.20(\mathrm{~s}, 6 \mathrm{H}$, triphenylene $\mathrm{CH}), 7.27-7.29(\mathrm{~m}, 20 \mathrm{H}), 7.40-7.48(\mathrm{~m}, 20 \mathrm{H}), 7.60-7.63$ $(\mathrm{m}, 13 \mathrm{H}))$ [10]. The hydrolysis of 3 resulted in the preparation of 2,3,6,7,10,11-hexaiminotriphenylene hydrochloric acid salts (80\%, DART MS $m / z 319\left[\mathrm{M}+\mathrm{H}^{+}\right.$; ${ }^{1} \mathrm{H}$ NMR (300 MHz, DMSO) 83.43 (brs, $18 \mathrm{H}), 7.85(\mathrm{~s}, 6 \mathrm{H})$ ). Finally, we obtained $\mathrm{Ni}_{3}(\mathrm{HITP})_{2}$ as black powders $(60 \%)$. The structural characterizations of each intermediate and $\mathrm{Ni}_{3}(\mathrm{HITP})_{2}$ were performed by NMR (JEOL JNM-AL300, Tokyo, Japan), mass spectroscopy (DART, JEOL JMS-Q1000TD; MALDI-TOF MS, Bruker Japan Autoflex II, Yokohama, Japan), transmission electron microscopy (JEOL JEM-3100FEF, Tokyo, Japan), X-ray diffraction (Rigaku RINT-TTR III/NM, Akishima, Japan), and X-ray photoelectron spectroscopy (XPS, ULVAC-PHI, Inc. PHI 5000 VersaProbe II, Chigasaki, Japan). DC electrical conductivity was measured using the 4-point probe method (Mitsubishi Chemical Loresta GP Model MCP-T610, Yamato, Japan). Thermoelectric voltage was recorded using a Seebeck coefficient measurement system (MMR technologies K20SB100-3R, San Jose, CA, USA) with a high-impedance amplifier [11-15]. Test specimens were prepared by soft-pressing at $18.5 \mathrm{MPa}(\sim 3$ ton). 


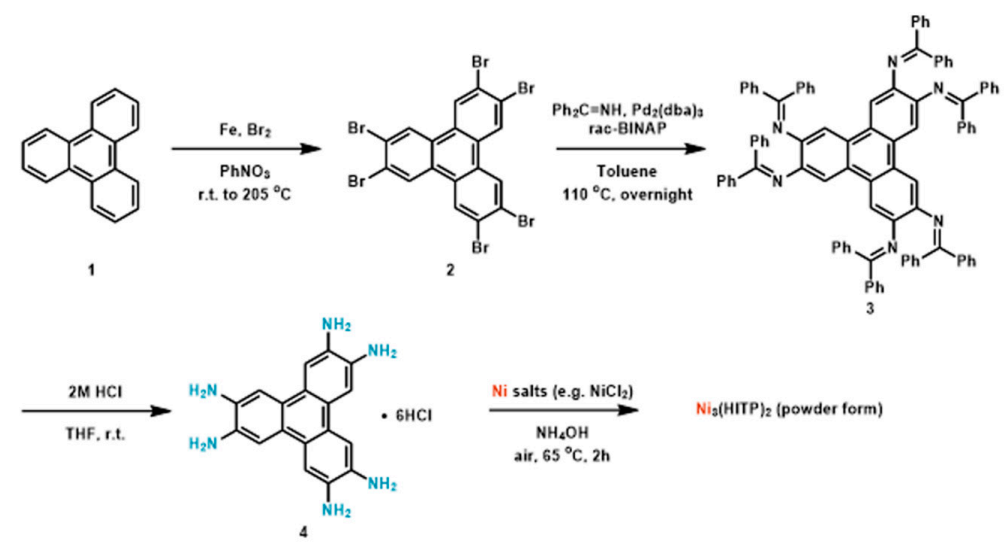

Scheme 1. A synthetic route to $\mathrm{Ni}_{3}(\mathrm{HITP})_{2}$.

\section{Results}

\subsection{Structural Characterization}

The XRD patterns of prepared powders indicated the highly ordered hexagonal lattice with intense (100), (200), and (001) peaks (Figure 2a-d). In detail, these structures were assigned to the eclipsed or slipped-parallel structures, or both, corresponding to the first report on their preparation [7]. We examined the effects of $\mathrm{Ni}$ salts species $\left(\mathrm{NiCl}_{2}, \mathrm{Ni}\left(\mathrm{NO}_{3}\right)_{2}, \mathrm{NiBr}_{2}\right.$, and $\left.\mathrm{Ni}(\mathrm{OTf})_{2}\right)$ on the crystallinity of final products. In the basic aqueous condition, $\mathrm{NiCl}_{2}$ was likely to afford sharp XRD patterns, indicating the most crystalline solids as discussed later. Such difference might result from the solubility and reactivity of $\mathrm{Ni}^{2+}$ feedstocks. TEM observation revealed the highly ordered mesoporous structures with hexagonal lattices (Figure 2e). The magnified image more clearly displays the hexagonally ordered mesopores (Figure 2f). Additionally, tens-nm-long mesochannel structures formed, indicating moderate c-axis stacking. The averaged pore diameter found in TEM images was approximately $2.0 \mathrm{~nm}$, and this size was consistent with the molecular modeling [7] (Figure 1b) and the (100) peak $(d$-spacing $=2.1 \mathrm{~nm})$ observed in the XRD profile (Figure 2a). In addition to such mesochannel bundles, the powders were also likely to consist of laterally grown nanosheets as the sheet edges were observed. This lateral growth is consistent with the strong (100) peak in the powder XRD. Further elemental analyses were performed using XPS (Figure S1).
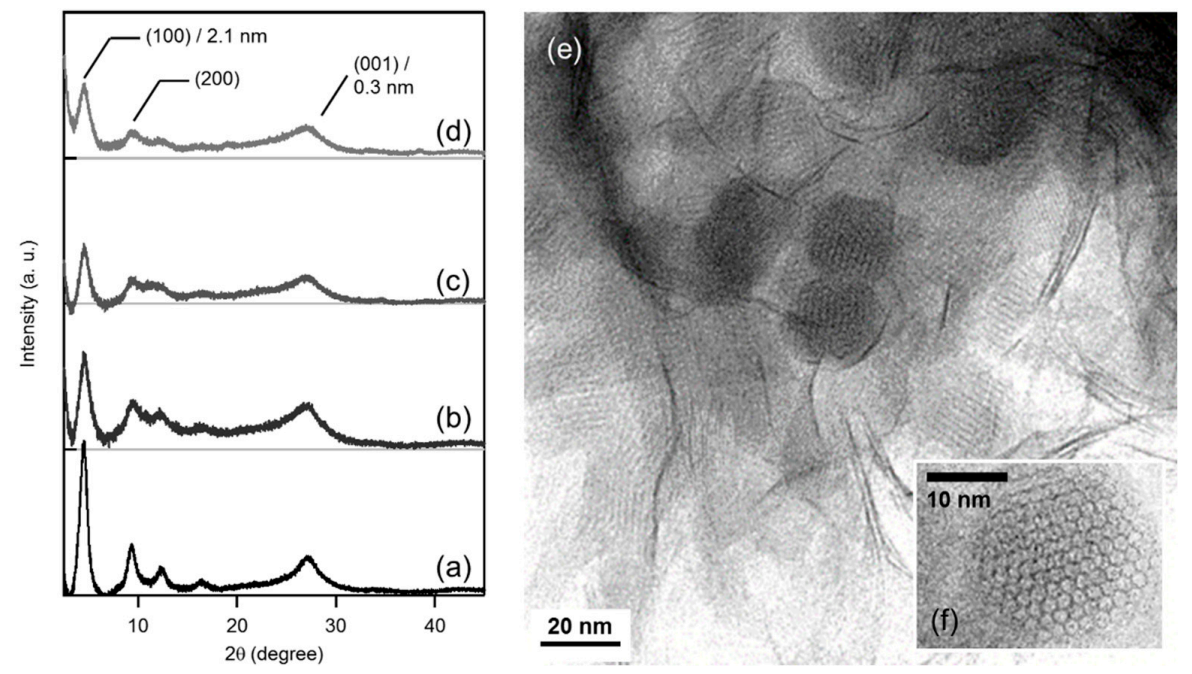

Figure 2. XRD patterns of $\mathrm{Ni}_{3}(\mathrm{HITP})_{2}$ powders prepared using (a) $\mathrm{NiCl}_{2}$, (b) $\mathrm{Ni}\left(\mathrm{NO}_{3}\right)_{2}$, (c) $\mathrm{NiBr}_{2}$, and (d) $\mathrm{Ni}(\mathrm{OTf})_{2}$. (e) Representative TEM images of $\mathrm{Ni}_{3}(\mathrm{HITP})_{2}$. An inset shows a magnified image. 
We prepared pellets by pressing the powder and evaluated the electrical conductivity of approximately $1 \mathrm{~S} \cdot \mathrm{cm}^{-1}$, which mostly reproduces the previous study [7]. Conduction pathway is attributed mostly to laterally grown framework, and in this work, such lateral electron transport in single crystals was limited within several tens nanometer. Therefore, an approach to promoting lateral crystal growth would be required for further improving electrical conductivity in the future work.

\subsection{Transport Study}

Soft-pressed pellets showed relatively low electrical resistance, which allowed to evaluate the Seebeck coefficient. The Seebeck coefficient is a generated voltage across the temperature gradient. For common metals, the relationship of the Seebeck coefficient $(\alpha)$ with electrical conductivity $(\sigma)$ can be expressed as,

$$
\alpha=\left.\frac{\pi^{2}}{3} \frac{k_{B}^{2} T}{q} \frac{1}{\sigma} \frac{\delta \sigma}{\delta \varepsilon}\right|_{\varepsilon=E_{F}}
$$

where $k_{B}$ is the Boltzmann constant, $q$ is the elemental charge, $T$ is the absolute temperature, and $E_{F}$ is the Fermi energy. This equation is known as the Mott formula, and simply indicates the trade-off relationship between $\sigma$ and $\alpha$ [16].

First, the Seebeck coefficients observed here were small $\left(|\alpha|<20 \mu \mathrm{V} \cdot \mathrm{K}^{-1}\right)$, suggesting the dominant contribution of metallic conduction in $\mathrm{Ni}_{3}(\mathrm{HITP})_{2}$. These values were indeed close to the typical Seebeck coefficient of metals [e.g., $-10 \mu \mathrm{V} \cdot \mathrm{K}^{-1}$ for palladium, and $+14 \mu \mathrm{V} \cdot \mathrm{K}^{-1}$ for iron]. Second, the sign of the Seebeck coefficient was negative, indicating n-type transport. Furthermore, we found the tunable thermoelectric properties of $\mathrm{Ni}_{3}(\mathrm{HITP})_{2}$ pellets dependent on salt species used in the synthesis. We used four nickel salts $\left(\mathrm{NiCl}_{2}, \mathrm{Ni}\left(\mathrm{NO}_{3}\right)_{2}, \mathrm{NiBr}_{2}\right.$, and $\left.\mathrm{Ni}(\mathrm{OTf})_{2}\right)$ as feedstocks, where $\mathrm{NiCl}_{2}$-derived $\mathrm{Ni}_{3}(\mathrm{HITP})_{2}$ pellets demonstrated the highest electrical conductivity $\left(\sim 0.7 \mathrm{~S} \cdot \mathrm{cm}^{-1}\right.$, Figure $\left.3 \mathrm{a}\right)$. As the electrical conductivity increased, the Seebeck coefficient decreased gradually. We further considered the effect of the crystalline size $(D)$, which is conventionally evaluated by the Scherrer's equation as follows,

$$
D=\frac{K \lambda}{B \cos \theta}
$$

where $B$ is the observed line width, $\lambda$ is the wavelength of incident $X$-ray, and $K$ is the Scherrer constant. We found that the large $D$ value indicating high crystallinity leads to an increasing in conductivity and a decreasing in the negative Seebeck coefficient as shown in Figure 3a. The analyses of a (100) peak with the Scherrer's equation revealed that the $D$ value can be modulated from $11 \mathrm{~nm}$ to $95 \mathrm{~nm}$, using the different $\mathrm{Ni}$ salt feedstocks (Figure $2 \mathrm{a}-\mathrm{d}$ ). The increasing $D$ value was then significantly influential to electrical conductivity, rather than the Seebeck coefficient. As a result, the $\mathrm{Ni}_{3}(\mathrm{HITP})_{2}$ pellet derived from $\mathrm{NiCl}_{2}$ exhibited the highest power factor $\left(\sigma \alpha^{2}\right)$ of $1.5 \times 10^{-8} \mathrm{~W} \cdot \mathrm{m}^{-1} \cdot \mathrm{K}^{-2}$ (Figure $3 \mathrm{~b}$ ) with the largest $D$ value. We could not perform further reliable chemical doping to the powdery samples since the pellets were fragile.

(a)

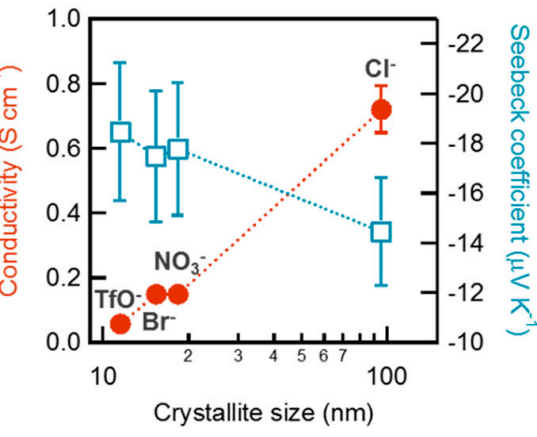

(b)

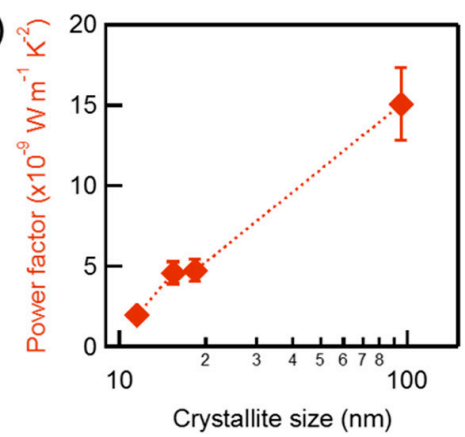

Figure 3. (a) Electrical conductivity, the Seebeck coefficient, and (b) power factor of $\mathrm{Ni}_{3}(\mathrm{HITP})_{2}$ pellets as a function of crystallite size. 


\section{Discussion}

Some previous systematic studies on micro-scale conductivity has revealed that the 2D-CPs including dithiolene-based CPs [17-20] showed the metal-like temperature dependence of electrical conductivity exceeding $100 \mathrm{~S} \cdot \mathrm{cm}^{-1}$. Particularly, copper complexes showed the small temperature dependence of electrical conductivity and very small Seebeck coefficients ranging from -4 to $-10 \mu \mathrm{V} \cdot \mathrm{K}^{-1}$, which suggested metallic conduction associated with the variable-range hopping mode [20]. On the other hand, $\mathrm{Ni}_{3}(\mathrm{HITP})_{2}$ nanosheets showed the positive temperature dependence of electrical conductivity, and this has been attributed to semiconductor-like transport [7]. Foster has predicted that the pristine $\mathrm{Ni}_{3}(\mathrm{HITP})_{2}$ is intrinsically a metal, but due to defect- and slipping-related grain boundaries, most bulk solids could show semiconductor-like transport properties [21]. Such the opened band-gap energy was estimated to be as large as $100 \mathrm{meV}$. Thermoelectric properties reflect the band structure at the Fermi level especially energy gradient of the density of state at the Fermi-level. Since deep doping is expected in the present polycrystalline pellets, the observed thermoelectric properties are likely to highlight the metallic characteristics of $\mathrm{Ni}_{3}(\mathrm{HITP})_{2}$, which would veil the practical semiconductor-like characteristics [7]. In this consideration the larger grain size seems to support higher conductivity with less charge carrier scattering. High crystallinity could suppress the Seebeck coefficient as a result of the broadened density-of-state (DOS) distribution. Additionally, $\mathrm{Ni}_{3}(\mathrm{HITP})_{2}$ showed n-type transport, which is consistent with the oxidative formation of representative $\mathrm{Ni}^{2+} / \mathrm{Ni}^{3+}$ mixed valence compounds from $\mathrm{Ni}^{2+}$ [22], and the formation of HITP anion radicals.

\section{Conclusions}

We have examined the thermoelectric properties of $\mathrm{Ni}_{3}(\mathrm{HITP})_{2}$ pellets dependent on crystallinity. The Seebeck coefficient ranged from -10 to $-20 \mu \mathrm{V} \cdot \mathrm{K}^{-1}$, which seemed to reflect the metallic characteristics of $\mathrm{Ni}_{3}(\mathrm{HITP})_{2}$. Crystallinity was crucial for exploiting high electrical conductivity, contributing to an increase in the thermoelectric power factor. The present work gives an insight into the design of molecular thermoelectric materials.

Supplementary Materials: The following are available online at http:/ / www.mdpi.com/2073-4360/10/9/962/s1, Figure S1: XPS of a $\mathrm{Ni}_{3}(\mathrm{HITP})_{2}$ powder.

Author Contributions: Conceptualization, Y.N. and T.K.; Methodology, D.S.; Formal Analysis, D.S.; Investigation, Y.N. and D.S.; Data Curation, Y.N. and D.S.; Writing-Original Draft Preparation, Y.N.; Writing-Review \& Editing, T.K.; Supervision, Y.N. and T.K.; Funding Acquisition, Y.N.

Funding: This research was funded by NEDO and JST PRESTO grant number JPMJPR16R6.

Acknowledgments: This work made use of NAIST common facilities supported by MEXT Nanotechnology Platform program.

Conflicts of Interest: The authors declare no conflict of interest.

\section{References}

1. Bisri, S.Z.; Shimizu, S.; Nakano, M.; Iwasa, Y. Endeavor of iontronics: From fundamentals to applications of ion-controlled electronics. Adv. Mater. 2017, 29, 1607054. [CrossRef] [PubMed]

2. Akamatu, H.; Inokuchi, H.; Matsunaga, Y. Electrical conductivity of the perylene-bromine complex. Nature 1954, 173, 168. [CrossRef]

3. Epstein, A.J.; Etemad, S.; Garito, A.F.; Heeger, A.J. Metal-insulator transition and antiferromagnetism in a one-dimensional organic solid. Phys. Rev. B 1972, 5, 952-977. [CrossRef]

4. Ferraris, J.; Cowan, D.O.; Walatka, V.; Perlstein, J.H. Electron transfer in a new highly conducting donor-acceptor complex. J. Am. Chem. Soc. 1973, 95, 948-949. [CrossRef]

5. Sun, Y.; Sheng, P.; Di, C.; Jiao, F.; Xu, W.; Qiu, D.; Zhu, D. Organic thermoelectric materials and devices based on $p$ - and $n$-type poly(metal 1,1,2,2-ethenetetrathiolate)s. Adv. Mater. 2012, 24, 932-937. [CrossRef] [PubMed] 
6. Cheng, X.-F.; Shi, E.-B.; Hou, X.; Shu, J.; He, J.-H.; Li, H.; Xu, Q.-F.; Li, N.-J.; Chen, D.-Y.; Lu, J.-M. 1D $\pi$-d conjugated coordination polymers for multilevel memory of long-term and high-temperature stability. Adv. Electron. Mater. 2017, 3, 1700107. [CrossRef]

7. Sheberla, D.; Sun, L.; Blood-Forsythe, M.A.; Er, S.; Wade, C.R.; Brozek, C.K.; Aspuru-Guzik, A.; Dincă, M. High electrical conductivity in $\mathrm{Ni}_{3}(2,3,6,7,10,11 \text {-hexaiminotriphenylene })_{2}$, a semiconducting metal-organic graphene analogue. J. Am. Chem. Soc. 2014, 136, 8859-8862. [CrossRef] [PubMed]

8. Sheberla, D.; Bachman, J.C.; Elias, J.S.; Sun, C.-J.; Shao-Horn, Y.; Dincă, M. Conductive MOF electrodes for stable supercapacitors with high areal capacitance. Nat. Mater. 2017, 16, 220-224. [CrossRef] [PubMed]

9. Yatabe, T.; Harbison, M.A.; Brand, J.D.; Wagner, M.; Rabe, J.P. Extended triphenylenes: Synthesis, mesomorphic properties and molecularly resolved scanning tunneling microscopy images of hexakis(dialkoxyphenyl)triphenylenes and dodeca(alkoxy)tris(triphenylenylene)s. J. Mater. Chem. 2000, 10, 1519-1525. [CrossRef]

10. Chen, L.; Kim, J.; Ishizuka, T.; Honsho, Y.; Saeki, A.; Seki, S.; Ihee, H.; Jiang, D. Noncovalently Netted, photoconductive sheets with extremely high carrier mobility and conduction anisotropy from triphenylene-fused metal trigon conjugates. J. Am. Chem. Soc. 2009, 131, 7287-7292. [CrossRef] [PubMed]

11. Nonoguchi, Y.; Ohashi, K.; Kanazawa, R.; Ashiba, K.; Hata, K.; Nakagawa, T.; Adachi, C.; Tanase, T.; Kawai, T. Systematic conversion of single walled carbon nanotubes into n-type thermoelectric materials by molecular dopants. Sci. Rep. 2013, 3, 3344. [CrossRef] [PubMed]

12. Nonoguchi, Y.; Nakano, M.; Murayama, T.; Hagino, H.; Hama, S.; Miyazaki, K.; Matsubara, R.; Nakamura, M.; Kawai, T. Simple salt-coordinated n-type nanocarbon materials stable in air. Adv. Funct. Mater. 2016, 26, 3021-3028. [CrossRef]

13. Nonoguchi, Y.; Tani, A.; Ikeda, T.; Goto, A.; Tanifuji, N.; Uda, R.M.; Kawai, T. Water-processable, air-stable organic nanoparticle-carbon nanotube nanocomposites exhibiting n-type thermoelectric properties. Small 2017, 13, 1603420. [CrossRef] [PubMed]

14. Nakano, M.; Nakashima, T.; Kawai, T.; Nonoguchi, Y. Synergistic Impacts of Electrolyte Adsorption on the Thermoelectric Properties of Single-Walled Carbon Nanotubes. Small 2017, 13, 1700804. [CrossRef] [PubMed]

15. Nonoguchi, Y.; Iihara, Y.; Ohashi, K.; Murayama, T.; Kawai, T. Air-tolerant fabrication and enhanced thermoelectric performance of $n$-type single-walled carbon nanotubes encapsulating 1,1'-bis(diphenylphosphino)ferrocene. Chem. Asian J. 2016, 11, 2423-2427. [CrossRef] [PubMed]

16. Mott, N.F.; Jones, H. The Theory of the Properties of Metals; Clarendon Press: Oxford, UK, 1936.

17. Hmadeh, M.; Lu, Z.; Liu, Z.; Gándara, F.; Furukawa, H.; Wan, S.; Augustyn, V.; Chang, R.; Liao, L.; Zhou, F.; et al. New porous crystals of extended metal-catecholates. Chem. Mater. 2012, 24, 3511. [CrossRef]

18. Kambe, T.; Sakamoto, R.; Hoshiko, K.; Takada, K.; Miyachi, M.; Ryu, J.-H.; Sasaki, S.; Kim, J.; Nakazato, K.; Takata, M.; et al. $\pi$-Conjugated nickel bis(dithiolene) complex nanosheet. J. Am. Chem. Soc. 2013, 135, 2462. [CrossRef] [PubMed]

19. Kambe, T.; Sakamoto, R.; Kusamoto, T.; Pal, T.; Fukui, N.; Hoshiko, K.; Shimojima, T.; Wang, Z.; Hirahara, T.; Ishizaka, K.; et al. Redox control and high conductivity of nickel bis(dithiolene) complex $\pi$-nanosheet: A potential organic two-dimensional topological insulator. J. Am. Chem. Soc. 2014, 136, 14357. [CrossRef] [PubMed]

20. Huang, X.; Sheng, P.; Tu, Z.; Zhang, F.; Wang, J.; Geng, H.; Zou, Y.; Di, C.-A.; Yi, Y.; Sun, Y.; et al. A two-dimensional $\pi$ - $d$ conjugated coordination polymer with extremely high electrical conductivity and ambipolar transport behavior. Nat. Commun. 2015, 6, 7408. [CrossRef] [PubMed]

21. Foster, M.E.; Sohlberg, K.; Allendorf, M.D.; Talin, A.A. Unraveling the semiconducting/metallic discrepancy in $\mathrm{Ni}_{3}(\mathrm{HITP})_{2}$. J. Phys. Chem. Lett. 2018, 9, 481-486. [CrossRef] [PubMed]

22. Kurahashi, T.; Fujii, H. One-electron oxidation of electronically diverse manganese(III) and nickel(II) salen complexes: Transition from localized to delocalized mixed-valence ligand radicals. J. Am. Chem. Soc. 2011, 133, 8307. [CrossRef] [PubMed]

(c) 2018 by the authors. Licensee MDPI, Basel, Switzerland. This article is an open access article distributed under the terms and conditions of the Creative Commons Attribution (CC BY) license (http:/ / creativecommons.org/licenses/by/4.0/). 